\title{
VAMP-8 gene variant is associated with increased risk of early myocardial infarction
}

Jarosław Gorący', Iwona Gorący², Mariusz Kaczmarczyk², Miłosz Parczewski³, Lech Cyryłowski, Mirosław Brykczyński ${ }^{5}$, Małgorzata Peregud-Pogorzelska', Andrzej Ciechanowicz²

1Department of Cardiology, Pomeranian Medical University, Szczecin, Poland

2Department of Laboratory Diagnostics and Molecular Medicine, Pomeranian Medical University, Szczecin, Poland

3Department of Infectious Diseases and Hepatology, Pomeranian Medical University, Szczecin, Poland

${ }^{4} \mathrm{CT}$ and MRI Clinic, Non-public Health Care Clinic "Our Doctor", Szczecin, Poland

${ }^{5}$ Department of Cardiosurgery, Pomeranian Medical University, Szczecin, Poland

Submitted: 28 May 2010

Accepted: 26 July 2010

Arch Med Sci 2011; 7, 3: 440-443

DOI: 10.5114/aoms.2011.23409

Copyright @ 2011 Termedia \& Banach

\section{Abstract}

Introduction: Single nucleotide polymorphism in the 3' untranslated region of the vesicle-associated membrane protein gene (VAMP-8) has been associated with increased risk of early-onset myocardial infarction (MI). In this study the risk of early onset MI conferred by VAMP-8 gene polymorphism was investigated in a group of 171 male subjects.

Material and methods: Male patients with a history of MI who underwent coronary angiography were enrolled and divided into early (incident $<55$ years of age) and late (incident $\geq 55$ years of age) MI onset groups. Apart from the RFLP-PCR based analysis of the VAMP-8 variant, history of hypertension, lipid abnormalities, smoking and body mass index were recorded. In statistical analyses odds ratios and relative risk were used as a measure of genotype-MI association while logistic regression was implemented for evaluation of MI risk factor strength.

Results: VAMP-8 A allele frequency proved to be significantly higher in the earlyonset $\mathrm{MI}$ group and conferred higher relative risk of early $\mathrm{MI}$ in the investigated cohort, when calculated for the individual A allele $(p=0.029)$. In logistic regression analyses no association between risk genotypes and traditional risk factors was observed.

Conclusions: In this study VAMP-8 A variant was identified as a risk allele for early $\mathrm{MI}$ in male subjects.

Key words: myocardial infarction, VAMP-8, gene polymorphism, risk factor.

\section{Introduction}

Ischaemic heart disease (IHD) and its most grave consequence, namely myocardial infarction (MI), remain among the most common public health threats worldwide. High and medium income countries are strongly affected, with the yearly rate of $\mathrm{MI}$ throughout Europe ranging from 90 to 312 per one hundred thousand individuals yearly and a high number of myocardial infarction episodes with ST elevation (STEMI). In Poland cardiovascular disease (CVD) remains a primary public health issue as well, being the leading cause of death (45.6\% in 2008). Among males below 59

\section{Corresponding author:}

Mariusz Kaczmarczyk MD, PhD

Department of Laboratory

Diagnostics

and Molecular Medicine

Pomeranian Medical University

Szczecin, Poland

E-mail:

mariush@sci.pam.szczecin.pl 
years of age CVD-associated mortality was threefold higher, with gender-related differences becoming insignificant in older individuals [1].

Extensive, multicentre research has identified an array of "classical" MI risk factors with age, smoking, hypercholesterolaemia, hypertension, type 2 diabetes and positive IHD family history being the most common [2]. It must be noted that genetic factors should also be considered, as indicated by family and gene variant association studies performed on large groups and high numbers of genetic markers [3-5]. Of these, high throughput single nucleotide polymorphism (SNP) studies resulting in the identification of risk-associated alleles proved to be of the highest value $[4,6]$, with results validated by risk-allele targeted association studies $[7,8]$.

For this study a genetic variant of a vesicleassociated membrane protein (VAMP), previously unstudied in the Polish population, was selected. Vesicle-associated membrane protein proteins play a crucial role in the regulatory mechanisms of platelet secretory function [9]. It has been demonstrated that cellubrevin (VAMP-3) and endobrevin (VAMP-8) form soluble N-ethylmaleimidesensitive factor attachment protein receptor (SNARE) complexes in human platelets and mediate granule secretion [10, 11]. VAMP-8 gene polymorphism in the 3' untranslated region (accession number rs 1010) has been identified as one of the risk factors for early-onset $\mathrm{MI}$ in a large case-control study analysing 11647 human SNPs [6]. Associations between VAMP-8 gene polymorphisms and in vitro platelet functions were studied, but did not give conclusive data on the polymorphismrelated mechanism of platelet dysfunction [12, 13].

The aim of this research was to analyse an association between risk of early onset of $\mathrm{MI}(<55$ years of age) among male subjects and VAMP- 8 gene polymorphism (rs 1010). This is the first study on Polish subjects and in the North-European area.

\section{Material and methods}

\section{Subjects}

The study protocol was approved by the local Ethical Committee with all patients voluntarily consenting to participate in the research.

For the study group 171 male patients (mean age of $55.7 \pm 9.8$ years) were enrolled. All cases had a history of $\mathrm{Ml}$ and underwent interventional procedures (coronary angiography) at the Department of Cardiology, Pomeranian Medical University, Szczecin. The group was divided into early-onset MI (infarction below the age of 55 years, $n=103$ ) and late onset MI subgroups (infarction at the age $\geq 55$ years, $n=68$ ). Resting ECG was performed in all cases; full medical and treatment history including smoking status was obtained and body mass index was calculated. Biochemical analyses in this group included determination of serum total cholesterol and triglyceride concentrations prior to the angiography. For genetic analyses whole blood samples were collected on EDTA anticoagulant. All subjects were treated according to the IHD treatment guidelines with ACE inhibitors, statin, $\beta$-blockers, and aspirin. The study protocol was approved by the local Bioethics Committee.

\section{Genotyping}

The QIAamp DNA Blood Mini Kit (QIAgen, Hilden, Germany) was used to extract genomic DNA from blood samples. The extraction was performed by following the manufacturer's protocol. DNA was resuspended in $200 \mu \mathrm{l}$ of AE buffer (QIAgen, Hilden, Germany) and stored at $4^{\circ} \mathrm{C}$ for further analyses. Identification of VAMP-8 A/G gene polymorphism was performed by a PCR method, previously described by Shiffman, using the following primers: sense: $5^{\prime}$ - GGG GGC TCC AAC TTT CTT CTC C and antisense $5^{\circ}$ - CTT TGC CAC TGG TGC CTT CTC TTA [6]. RFLP of the PCR product with the restriction enzyme Mae II was performed; for the A allele the final product of 494 base pairs (b.p.) remained undigested, while the $G$ variant gave digested products of 328 b.p. and 166 b.p. Products were electrophoresed in agarose gel stained with ethidium bromide and analysis results were recorded digitally.

\section{Statistical analysis}

Allele and genotype frequencies among cases and controls were compared with values predicted by the Hardy-Weinberg equilibrium. Odds ratios (OR) were calculated as a measure of association between genotype and MI. For each OR, we calculated the 2 -tailed probability value and $95 \% \mathrm{Cl}$. Odds ratios and $p$ values were calculated separately for $A$ and $G$ alleles.

A value of $p<0.05$ was considered as indicative of a statistically significant effect. Logistic regression models were designed to evaluate the importance of risk factors in $\mathrm{MI}$.

\section{Results}

Clinical and laboratory characteristics of early and late-onset $\mathrm{MI}$ subgroups are presented in Table I. Power of the analysis was supported by the strong statistical difference observed for the age criterion, applied after division into the subgroups. Additionally, lipid concentrations were higher in the early-onset MI group, while a notably higher number of hypertensive individuals was observed in the late onset MI group. No significant differences 
Table I. Baseline clinical and laboratory characteristics of study patients

\begin{tabular}{|lccc|}
\hline Variable & Ml $<55$ & MI $\geq 55$ & Value of $p$ \\
\hline Age of MI & $47.3 \pm 5.2$ & $63.4 \pm 6.2$ & 0.001 \\
\hline Hypertension [\%] & 44 & 60 & 0.033 \\
\hline Smokers [\%] & 61 & 54 & 0.380 \\
\hline $\begin{array}{l}\text { Body mass index } \\
{\left[\mathrm{kg} / \mathrm{m}^{2} \text { ] }\right.}\end{array}$ & $27.2 \pm 3.4$ & $27.0 \pm 3.6$ & 0.667 \\
\hline $\begin{array}{l}\text { Total cholesterol } \\
\text { [mg/dl] }\end{array}$ & $230 \pm 40$ & $218 \pm 39$ & 0.056 \\
\hline Triglycerides [mg/dl] & $201 \pm 157$ & $158 \pm 74$ & 0.035 \\
\hline
\end{tabular}

concerning smoking or body mass index were observed between the subgroups.

Allele and genotype distributions for both subgroups are presented in Table II. Distribution of individual $A A, A G$ and $G G$ genotypes did not differ significantly between the subgroups, while A allele frequency was significantly higher among subjects with early-onset MI (below the age of 55 years) when compared to late-onset MI (above 55 years). Relative risk (RR) of early-onset $\mathrm{MI}$ was demonstrated to be higher in individuals carrying the A allele. RR was calculated for both the individual A allele (difference statistically significant, $p=0.029$ ) and A allele containing genotypes (statistical differences not significant); in our study A was proven to be an early-MI risk associated allele.

Additionally, diagnosis of hypertension, smoking status, BMI, total cholesterol and triglyceride levels were correlated by logistic regression with frequency of the investigated alleles and genotypes, but no association between risk genotypes and traditional risk factors was observed.

\section{Discussion}

In this study the hypothesis that VAMP-8 rs1010 single nucleotide polymorphism is associated with increased risk of early-onset myocardial infarction was tested. This SNP was selected after a review of existing research data, in which it has been defined as one of the risk alleles for coronary heart disease, early-onset $\mathrm{MI}$ and ischaemic stroke in Caucasian populations $[3,7]$. The decision to limit our study to male cases only was based on the genetic risk analysis performed in the study by Shifmann to maximize the possibility to obtain a valid result [6]. Moreover, cardiovascular disease incidence among young men is higher than among young women and therefore genetic risk factor identification is of higher importance in this group.

Influence of the VAMP-8 rs1010 variants was proven to be additive, and was included in a coronary heart disease genetic risk score model for the Atherosclerosis Risk in Communities study [14]. As most of the studies so far have been performed in the United States, our study aimed to confirm the risk association described above in the Polish population, which could be representative of North-European populations. Extensive genetic research has been published in the field of genetic factors associated with early-onset coronary artery disease $[15,16]$, but precise identification of single nucleotide risk variants remains infrequent.

VAMP-8 rs1010 SNP was associated with earlyonset $\mathrm{MI}$ in our study, with the A variant identified as the risk allele. It must be noted that such a finding is discordant with the data published before, where the $G$ allele was indicated to be associated with enhanced risk in the large-scale SNP genotyping study performed by Shifmann in order to identify MI early-onset genetic risk factors [6]. That study was performed on a group of American Caucasians with $\mathrm{Ml}$ diagnosed at age < 55 among male subjects and < 60 for females; the odds ratio (OR) related to the disease was 1.26 for the $G$ risk allele for the entire group, while the estimated risk for this variant was even higher for males (OR 1.97). However, this analysis was unadjusted for age and diabetes. Additionally, in the aforementioned Atherosclerosis Risk in Communities study a high genetic risk score, defined as the presence of five single nucleotide polymorphisms including VAMP-8 rs1010, was associated with increased risk of incident coronary heart disease (CHD) after adjusting for traditional

Table II. VAMP-8 polymorphism associated risk of myocardial infarction in Polish males

\begin{tabular}{|c|c|c|c|c|c|c|}
\hline Genetic variant & $\mathrm{Ml}<55, n(\%)$ & $\mathrm{MI} \geq 55, n(\%)$ & $\mathrm{OR}_{\mathrm{A}}(95 \% \mathrm{Cl})$ & Value of $p$ & $\mathrm{OR}_{\mathrm{G}}(95 \% \mathrm{Cl})$ & Value of $p$ \\
\hline$A A+A G$ & $93(90.3)$ & $57(83.8)$ & $1.63(0.63-4.23)$ & 0.309 & - & - \\
\hline AA & $49(47.6)$ & $21(30.9)$ & $2.24(0.78-6.41)$ & 0.128 & 1 & - \\
\hline$A G$ & $44(42.7)$ & $36(52.9)$ & $1.37(0.50-3.75)$ & 0.531 & $0.66(0.32-1.35)$ & 0.247 \\
\hline GG & $10(9.7)$ & $11(16.2)$ & 1 & - & $0.45(0.16-1.28)$ & 0.128 \\
\hline$A G+G G$ & $54(52.4)$ & 47 (69.1) & - & - & $0.60(0.31-1.18)$ & 0.136 \\
\hline$A$ & $142(68.9)$ & $78(57.4)$ & $1.65(1.03-2.65)$ & 0.029 & - & - \\
\hline $\mathrm{G}$ & $64(31.1)$ & $58(42.6)$ & - & - & $0.61(0.38-0.97)$ & 0.029 \\
\hline
\end{tabular}

All findings concerning genotypes were adjusted for BMI, smoking, hypertension, cholesterol and triglyceride concentrations 
risk factors. In this study an association between the $\mathrm{G}$ risk allele and myocardial infarction was also confirmed $[3,14]$. These association analyses were performed for white populations, but no variable of early-onset MI was analysed.

On the other hand, van der Net et al., in an association study performed on patients with familial hypercholesterolaemia, did not confirm the findings of increased risk related to the VAMP-8 rs1010 variants [8]. This study was performed on relatively young (mean age of 48.8 years) Caucasian Europeans and might indicate a possible association between coronary heart disease and MI risk with this SNP.

It must also be noted that the sample analysed in our study is a Caucasian population from the north-western region of Poland, which due to the post-war migration is highly likely to be genetically diverse. The VAMP-8 allelic frequency is therefore highly likely to be similar in the whole of Poland. A strong influence of classical, environmental CVD and $\mathrm{MI}$ risk factors in the Polish population has been previously confirmed [17] but this genetic, riskallele based study contributes to the knowledge concerning the genetic epidemiology of CAD.

A limitation of the study is related to the fact that the occurrence of classical risk factors in the Polish population is high and thus they may play a greater role in atherosclerosis pathogenesis than genetic factors. However, further gene association studies are necessary for younger populations, with shorter time of exposure to Ml-promoting environmental factors, such as smoking, diet or obesity, where genetic risk factors would be of even greater influence and importance.

In conclusion, VAMP-8 rs1010 gene variants are related to the risk of early-onset myocardial infarction, with further gene association studies necessary for confirmation of its exact influence on coronary artery disease.

\section{Acknowledgments}

The study was financed by internal funding of the Pomeranian Medical University, Szczecin, Poland. The authors declare no conflict of interest with respect to this research.

\section{References}

1. Central Statistical Office. Life Expectancy Tables of Poland. 2008. Available on-line (www.stat.gov.pl).

2. Dolder MA, Oliver MF. Myocardial infarction in young men. Study of risk factors in nine countries. Br Heart J. 1975; 37: 493-503.

3. Morrison AC, Bare LA, Chamblese LE, et al. Prediction of coronary heart disease risk using a genetic risk score: the Atherosclerosis Risk in Communities Study. Am J Epidemiol 2007; 166: 28-35.

4. Shiffman D, O Meara E, Bare LA, et al. Association of gene variants with incident myocardial infarction in the cardiovascular health study, Arterioscler Thromb Vasc Biol 2008; 28: 173-9.

5. Wang Q, Rao S, Shen GQ, et al. Premature myocardial infarction novel susceptibility locus on chromosome 1P3436 identified by genome wide linkage analysis. Am J Hum Genet 2004; 74: 262-71.

6. Shiffman D, Rowland CM, Louie JZ, et al. Gene variants of VAMP8 and HNRPUL1 are associated with early-onset myocardial infarction. Arterioscler Thromb Vasc Biol 2006; 26: 1613-8.

7. Luke MN, Kane JP, Liu DM, et al. A polymorphism in the protease-like domain of apolipoprotein(a) is associated with severe coronary artery disease. Arterioscler Thromb Vasc Biol 2007; 27: 2030-6.

8. van der Net JB, Oosterveer DM, Versmissen J, et al. Replication study of 10 genetic polymorphisms associated with coronary heart disease in a specific high-risk population with familial hypercholesterolemia. Eur Heart J 2008; 29: 2195-201.

9. Polgar J, Chung SH, Reed GL. Vesicle-associated membrane protein 3 (VAMP-3) and VAMP-8 are present in human platelets and are required for granule secretion. Blood 2002; 100: 1081-3.

10. Graham GJ, Ren Q, Dilks JR, Blair P, Whiteheart SW, Flaumenhaft R. Endobrevin/VAMP-8-dependent dense granule release mediates thrombus formation in vivo. Blood 2009; 114: 1083-90.

11. Antonin W, Holroyd C, Tikkanen R, Höning S, Jahn R. R-SNARE endobrevin/VAMP-8 mediates homotypic fusion of early endosomes and late endosomes. Mol Biol Cell 2000; 11: 3289-98.

12. Ren Q, Barber HK, Crawford GL, et al. Endobrevin/Vamp8 is the primary $\mathrm{V}$-SNARE for the platelet release reaction, Mol Biol Cell 2007; 18: 24-33.

13. Gaussem P, Ishida BY, Fontana P, et al. No influence of the VAMP8 Rs1010 single nucleotide polymorphism on platelet functions in vitro. J Cell Mol Med 2008; 12: 1-3.

14. Bare LA, Morrison AC, Rowland CM, et al. Five common gene variants identify elevated genetic risk for coronary heart disease. Genet 2007; 9: 682-9.

15. BHF Family Heart Study Research Group. A genomewide linkage study of 1,933 families affected by premature coronary artery disease. The British Heart Foundation (BHF) Family Heart Study. Am J Hum Genet 2007; 77: 1011-20.

16. Pajukanta P, Cargill M, Vitanen L, et al. Two loci on chromosomes 2 and $\mathrm{X}$ for premature coronary heart disease identified in early- and late-settlement population of Finland. Am J Hum Genet 2000; 67: 1481-93.

17. Dudek D, Siudak Z, Kuta M, et al. Clinical characteristics and treatment strategies in patients with acute coronary syndromes in hospitals without an on-site invasive facility. The Registry of Acute Coronary Syndromes in the Malopolska Region 2002-2003. Post Kardiol Inter 2005; 1: 97-106. 